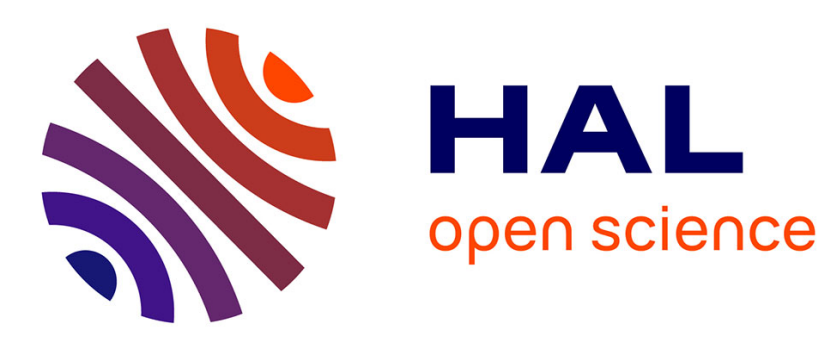

\title{
A physical model of turbulent fluids
}

Annie Luciani, Arash Habibi, Alexis Vapillon, Yvan Duroc

\section{To cite this version:}

Annie Luciani, Arash Habibi, Alexis Vapillon, Yvan Duroc. A physical model of turbulent fluids.

Eurographics 1995, Sep 1995, Maastricht, Netherlands. pp.16-29. hal-00484288

\section{HAL Id: hal-00484288 \\ https://hal.science/hal-00484288}

Submitted on 8 Apr 2014

HAL is a multi-disciplinary open access archive for the deposit and dissemination of scientific research documents, whether they are published or not. The documents may come from teaching and research institutions in France or abroad, or from public or private research centers.
L'archive ouverte pluridisciplinaire HAL, est destinée au dépôt et à la diffusion de documents scientifiques de niveau recherche, publiés ou non, émanant des établissements d'enseignement et de recherche français ou étrangers, des laboratoires publics ou privés. 


\title{
A Physical Model Of Turbulent Fluids
}

\author{
A. Luciani, A. Habibi, A. Vapillon, Y. Duroc \\ ACROE - LIFIA \\ INPG - 46 avenuc Félix Viallet \\ 38031 Grenoble cedex - FRANCE \\ Tél : (33) 76574669 - Fax : (33) 76574602 \\ c-mail : acroe(aimag.fr
}

\begin{abstract}
Turbulent phenomena are a subject of great interest for the computer graphic: community as well as for the physics community. In computer graphics, curren models of turbulent flow are mostly kincmatic and stochastic models. The model: presented in this paper are all physically-based and totally deterministic. They wer achieved using the Cordis-Anima physical modeller-simulator, which is based upor point physics connected by physical interactions. In order to obtain physically and visually fine phenomena, while using a rather low number of particles, we resort to multi-scale modelling : the turbulent phenomena are modelled by a medium-scalc physical model, whereas the refinement is achicved by a small-scale linear physical model. The final simulation is achieved by coupling these models. The resulting simulations present various phenomena inherent in turbulent fluids (curls, vortices. dissipation, diffusion). We also succeeded in reproducing several specific observed phenomena such as Kelvin-Helmholı and Von Karman turbulences.
\end{abstract}

\section{Discrete vs continuous models}

There are mainly two types of approaches for the modelling of physical phenomena : the continuous approach and the discrete approach. As shows [Gre 73], in classical modelling (figure 1):

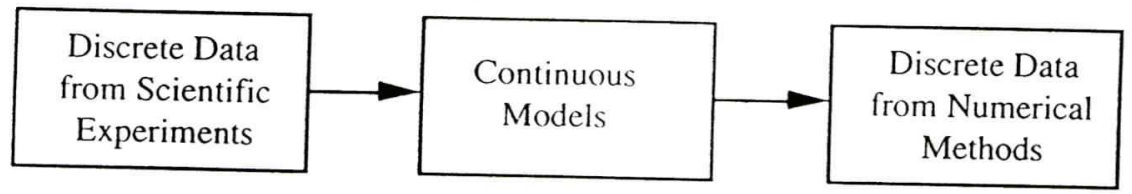

figure 1 the classical scientific procedure - [Gre 73]

continuous models can be directly replaced by discrete models to great advantage enabling the study of unpredictable or highly non-linear phenomena, for which the continuous formal equations are difficult to solve. This is all the more true for modelling the dynamic behaviour of non-stationary turbulent or chaotic systems such as fluids in non-laminar flow, smoke, propagation in complex environments (organic tissues, superconductors, porous substrata). All produce turbulent phenomena in which structured shapes such as vortices spontaneously appear, maintain themselves and possibly disappear; In these systems energetic dynamics play an important role. Vortices, curls, dissipation, diffusion and other turbulences appear as consequences of these very energetic exchanges. 


\section{Previous works}

\subsection{Motion modelling}

In the field of computer graphics, the turbulent phenomena in smoke and in flames are often modelled by superimposing a velocity field and physical objects. In [MP 89] and [LJCFR91], viscous flow has was modelled, but no turbulences were observed. In [WH 91] and in [CMTM 94] physical particles are carried by a fluid which is often represented by a kinematically defined velocity field. This is why the relation between the fluid and the particles is one-way (fluid $\rightarrow$ particle). The resulting animations represent deformable leaves moving in turbulent wind field in [WH 91], flames and their spreading in [PP 94] and the visual simulation of fire in [CMTM 94].

In [SF 93] the velocity field has one large-scale field determined as in [WH 91] and one small-scale field which corresponds to a filtered white noise. But the velocity of the particles is directly determined kinematically by the value of the velocity field at that point. This results in the simulation of turbulent phenomena such as smoke and vapour.

\subsection{Shape modelling}

When one uses particle modelling in the aim to obtain an image, the various particles that compose the object must appear as one continuous stretch of matter. This implies that each particle be replaced by an elementary volume.

[INA 90] considers that each particle is the center of a time-independent spherical density function. The density functions add up and form a global density field that is volume-rendered. Thus the elementary shapes mentioned above do not depend on the motion of the particles. In [SF 93], the density functions are time-dependent. Diffusion and dissipation are modelled by the variation in size and in value of each density function according to a predetermined law. In [PP 94], each particle is associated with a Gouraud shaded regular hexagon. The shape of these polygons changes according to an explicitly time-dependent law. In [CMTM 94] as well as in [PP 94], each particle leaves on the final image, a trace that represents its successive positions in the course of time. This is the "long exposure" method. In this case, the elementary shape associated with each particle depends also on its motion.

\subsection{Our aim}

Our aim is to obtain a generic physical model of these phenomena that may produce a great variety of different behaviours and, in particular, react to unpredicted external physical events. Therefore, the kinematic or semi-kinematic models are not adequate. The model must be totally dynamic. We use a discrete model based upon point physics. In view of the importance of the energetic interactions in these phenomena we will take into account the physical interactions between the particles but also the actions from the environment on the particles, and the feedback (from the particles on the environment). This is a closed-loop interaction.

As for shape modelling, we extend this to the notion of refinement. The shape is also generated by physical modelling in order to obtain a deformable shape around each matter point. This deformation must not be arbitrary. It must be coherent with the physical properties (e.g. diffusion and dissipation) of the global model. In this paper it is modelled by a local physical model already described in [LHM 95] in accordance with the reference phenomena that are to be reproduced and in accordance with the physical behaviour of the particles. 


\section{Cordis - Anima modeller-simulator}

Cordis-Anima is a physical modeller-simulator based on point physics [CLF 93]. It is a well-proven system on which all of our previous works were carried out. It is based on the construction of physical models by the assembly of a possibly large number of a very limited set of automata. These automata are divided in two types : mass elements and interaction elements. The input of the former is a force value and the output is a position. The input of the latter is two positions and the output is two opposite forces. The first type is characterized by only one algorithm (Newton's second law). The second type is characterized by linear or piecewise linear, or discontinuous elastic or viscous interaction functions. All of our models are constructed by the assembly of these algorithms in networks in which the nodes are mass automata and arcs are interaction automata.

\section{The viscous interaction hypothesis : theoretical aspects}

\subsection{Viscosity as an emergent phenomenon - the mixing layer}

Lct us consider a set of particles, or a fluid at a microscopic level, in which motion is composed of thermal motion and a global motion called convection. If there is a velocity gradient between two zones of such a fluid, they exchange momentum at the junction of both zones by particle diffusion, by molecular collisions. Thus, the zone with higher velocity loses momentum and the slower fluid gains momentum. At a macroscopic level, these collisions define an emergent shear force proportional to the velocity gradient. The proportionality coefficient is the dynamic viscosity. The junction between these zones is an interface called the mixing layer. (figure 2) This layer is unstable and may go into oscillation and thus trigger whirlpools [Les 94].

\subsection{Viscous particle model}

This interaction phenomenon between two zones of molecular particles may be modelled by a set of particles linked to a substratum by a viscous interaction. The involved particles are not at a molecular scale, but at a scale sufficient for the representation of viscous resistance to flow (figure 2 ).

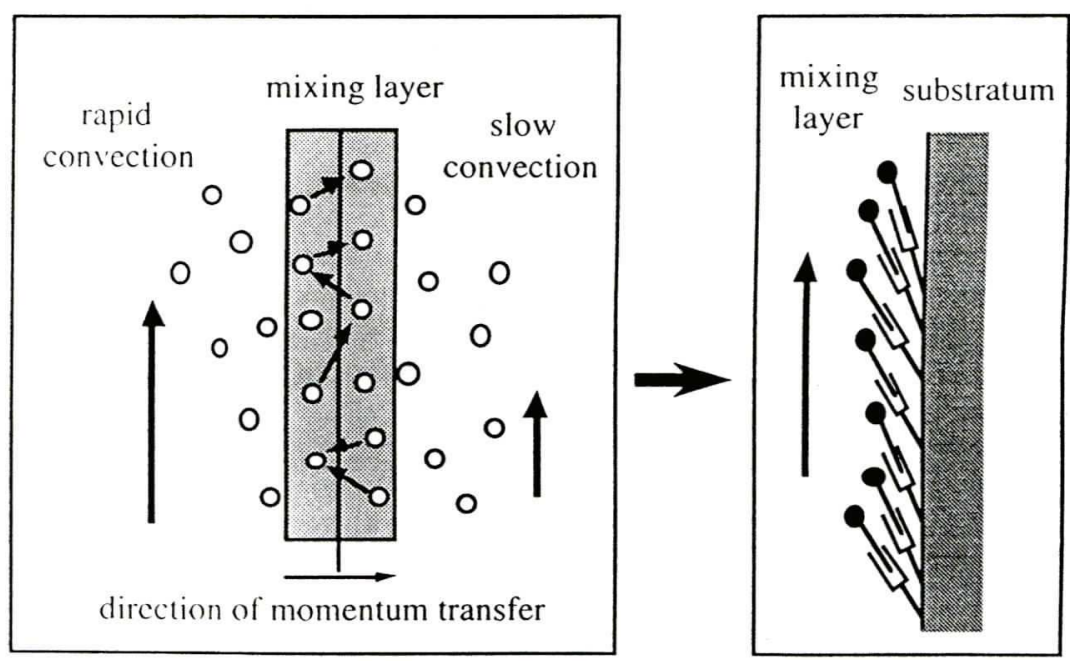

figure 2 Dynamic viscosity accounts for the collisions in the mixing layer 
This is a discrete physics particle implementation of the "newtonian fluid" model. It represents the Newton's third law : "The resistance occurring within a fluid is proportional to the velocity at which the different elements of the fluid move apart from each other". The Navier-Stokes equation is the formalization of this very law. More generally, in the mixing layer, viscosity is always higher than in other zones. The simplest particle model able to account for this phenomenon is composed of a set of particles in two-state viscous interaction with each other and with a substratum. The interaction law is described below :

$$
\begin{array}{lll}
\mathrm{F}_{\mathrm{pp}}=\mathrm{Z}_{\mathrm{pp}} \cdot \mathrm{Vr}_{\mathrm{pp}} & \mathrm{F}_{\mathrm{ps}}=\mathrm{Z}_{\mathrm{ps}} \cdot \mathrm{Vr}_{\mathrm{ps}} \\
\text { If } \mathrm{D}_{\mathrm{pp}}<\mathrm{T}_{\mathrm{pp}} & \mathrm{Z}_{\mathrm{pp}}=\mathrm{Z}_{1} & \text { else } \mathrm{Z}_{\mathrm{pp}}=\mathrm{Z}_{2} \\
\text { If } \mathrm{D}_{\mathrm{ps}}<\mathrm{T}_{\mathrm{ps}} & \mathrm{Z}_{\mathrm{ps}}=\mathrm{Z}^{\prime}{ }_{1} & \text { else } \mathrm{Z}_{\mathrm{ps}}=\mathrm{Z}_{2}{ }_{2}
\end{array}
$$

where $F_{p p}\left(\right.$ resp $\left.F_{p s}\right)$ are the inter-particle (resp. particle-substratum), forces $\mathrm{D}_{\mathrm{pp}}\left(\right.$ resp. $\mathrm{D}_{\mathrm{ps}}$ ) are the inter-particle (resp. particle - substratum) distances $\mathrm{Vr}_{\mathrm{pp}}$ (resp. $\mathrm{Vr}_{\mathrm{ps}}$ ) : relative velocities of the particles (resp. of particle - substratum) Tpp (resp Tps) is the threshold of the inter-particle (resp particle-substratum) interaction

In what follows, this type of interaction will be called thresholded viscous interaction, and the value of $Z_{2}$ and $Z_{2}^{\prime}$ will be zero.

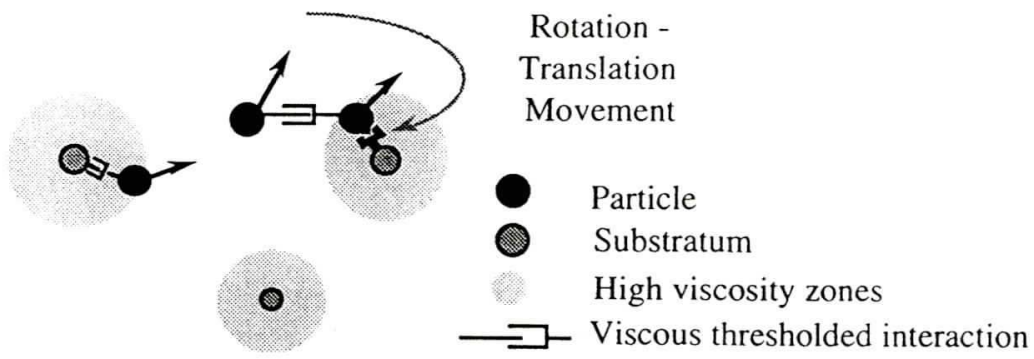

figure 3 : The generation of translation-rotation movements

When two masses $\left(\mathrm{P}_{1}, \mathrm{P}_{2}\right)$ are linked $\left(\mathrm{D}_{\mathrm{pp}}<\mathrm{T} \mathrm{pp}\right)$, and one of them $(\mathrm{P} 1)$ enters the high viscosity zone of a substratum mass (Dps<Tps) while P2 remains free, then $\mathrm{P} 1$ is slowed down. The velocity difference between P1 and P2 causes translation-rotation movements which are the basis phenomena that prime curls and whirlpools (figure 3 ).

The substratum may also be deformable. These deformations can be modelled by a mass-spring-damper network. The energy exchange goes in both directions (particles $\leftrightarrow$ substratum). In this way, the motion of the substratum can influence the motion of the particles, i.e. play the role of perturbation and bring the jet of active particles in oscillation and thus, trigger and intensify whirlpools. 
3.3. Experiments : curls, whirlpools, and Kelvin-Helmholtz turbulences in the discrete viscous model

A first model is composed of a jet of particles in thresholded viscous interactions with each other and with a deformable substratum. These particles flow out of a particle injector represented by two large circles at the bottom of figure 4 . The substratum is composed of a grid of masses linked by visco-elastic interactions. The position of the jet is not symmetrical in comparison with the grid. When the jet is fired on this substratum it produces curls, whirlpools, heaps, fractures, with dissipation.

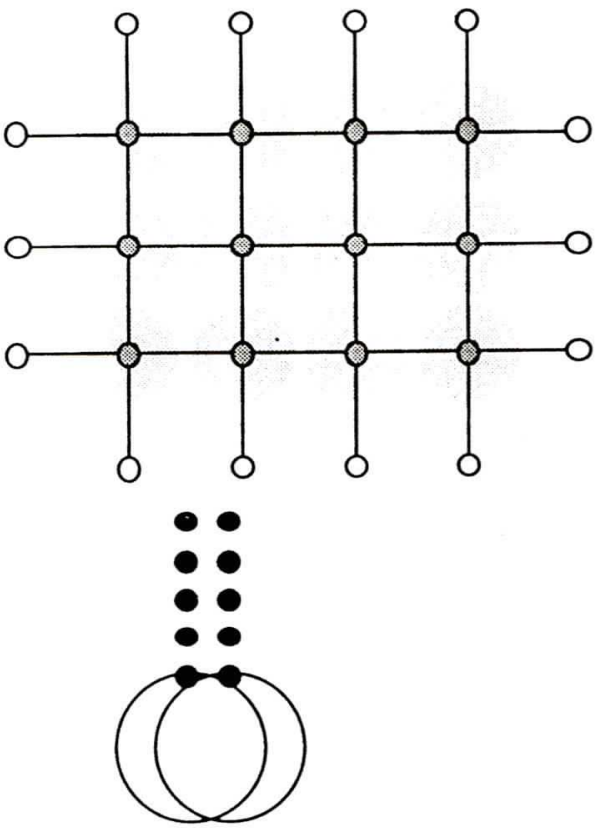

figure 4 A flow of particles fired in an ambient medium or substratum composed of visco-elastically attached points
On figure 4 the jet is represented in black and the substratum in grey. We obtain simulations in which we observe: (1) the deformation of the medium (represented by the larger circles), (2) the curls of the flowing particles (represented by points), (3) the dissipation of the flow, (4) its dispersion and (5) the final accumulation on the walls

Different behaviours were observed for different values of the visco-elasticity of the substratum, and the radius of influence of the local viscosities. For the same parameters of the substratum and the flow, the main parameter which determines the nature of the resulting phenomenon is the initial velocity of the flow. For very high velocities, the flow passes through the substratum with very low oscillations. For very small velocities, the flow is dissipated as soon as it enters the substratum.

Figures 5 to 8 show the variety of

behaviours that can be obtained simply by the variation of physical parameters. Each figure corresponds to two instants in the same simulation. The tested parameters are respectively: the ambient tension in the substratum.(figure 5), the initial velocity of the active particles (figure 6), the size of the substratum in terms of number of points (figure 7), and confinement (figure 8).

\subsubsection{Double-layer whirlpools}

The previous model in which the substratum had a very constrained movement is not general enough to produce other typical profiles of turbulent phenomena such as Kelvin-Helmholtz eddies. A much more general model of the Newton's third law is composed of two sets of particles in thresholded viscous interaction. 


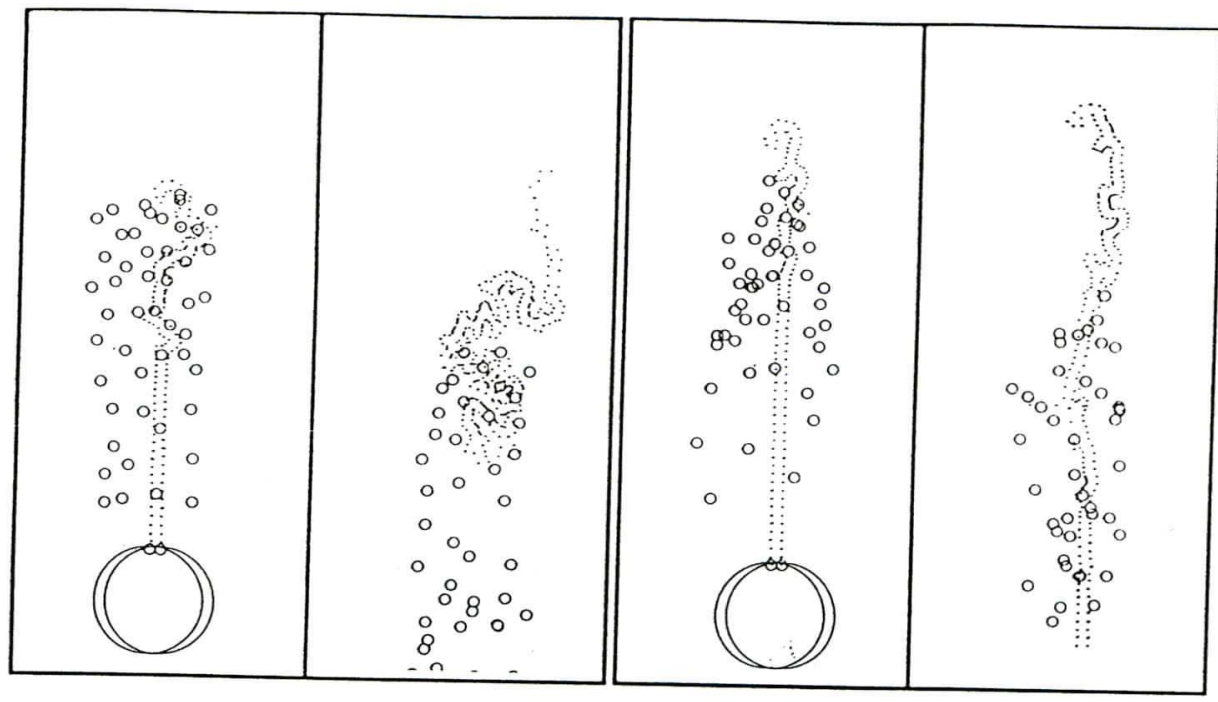

figure 5 Looser substratum

figure 6 Flow with greater initial velocity

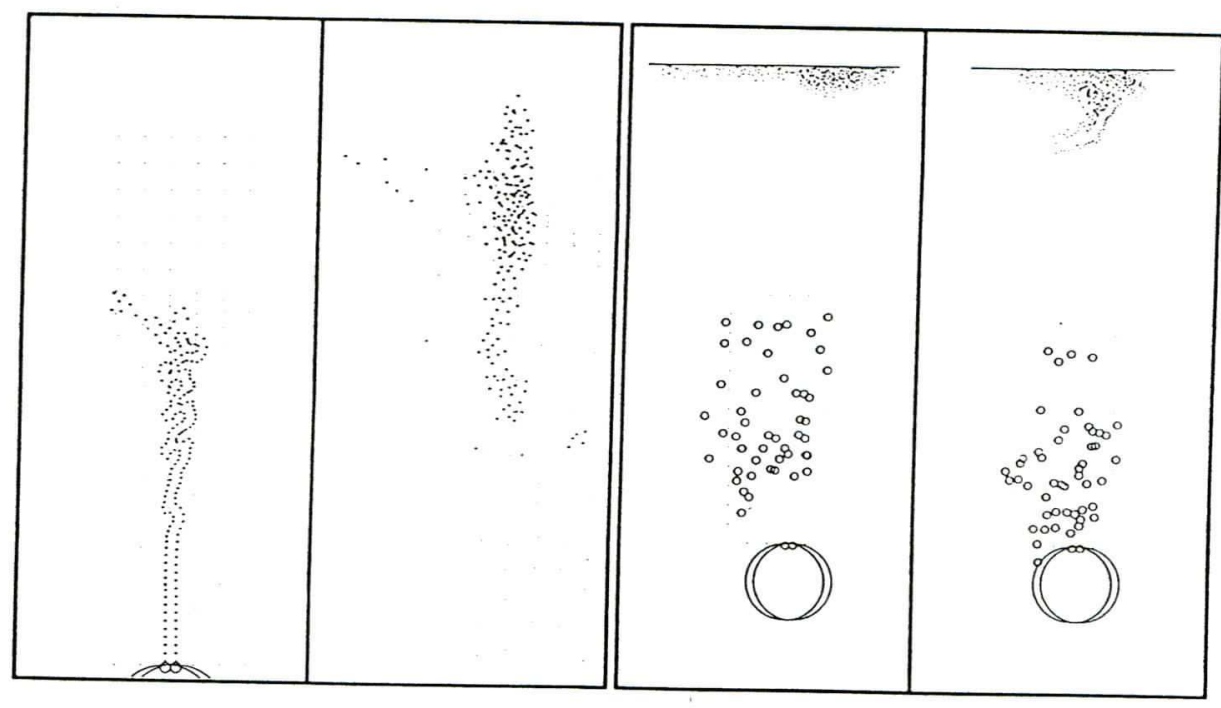

figure 7 Larger substratum

figure 8 Confinement

Let there be two fluids with different convection velocities V1 and V2 (figure 2.a). We can consider with no loss of generality that the velocities are opposite. We obtain this configuration by placing two sets of particle injectors on each side of the scene, facing each other and vertically shifted, in such a way that the two produced jets move along each other in opposite directions (figures 9,10 and 13) The gap between the opposite jets is chosen in order that the jets do not collide and thus avoid a front effect which is in contradiction to the mixing layer effect. When the flow of the two jets has reached a steady regime, we disymmetrically introduce a perturbation composed of one punctual mass in elastic buffer interaction with the fluids. 
Figure 9 shows the resulting porperly formed Kelvin-Helmholtz whirlpools in the case of thin mixing layers, obtained with two particle jets of two layers each.

On this figure, the perturbation is introduced between image $b$ and $c$. The particles of both jets cling to each other by viscosity and thus start a Kelvin-Helmholtz eddy. Then both vertically shifted and opposite jets exert a torque on the eddy. This is vorticity. The most central particles of the mixing layer coil first. Afterwards, as the eddy grows larger it propagates to outer layers.

\subsubsection{Multi-layer whirlpools}

Now let us experiment whether the eddy propagates in the fluid. This requires thicker jets. The same whirling phenomenon is observed. In addition, the eddy grows until the whole mixing layer is rolled. It can be noted that all particles do not roll in the eddy. In the previous experiment, all particles were involved, which produced one eddy alone in an empty space. Unlike this previous experiment, it can be observed that the flow is not stopped by the eddy. In view of the thickness of the simulated fluid and the shape of the eddy at the end of the simulations, we can assume that it would grow in the same manner in an infinite simulated environment.

The test parameters were : the inter-particle distance at injection, the gap between each jet, the injection velocity and frequency and the value of the viscous interactions.

The movement may remain global or divide depending on the viscosity. For very small viscosities, there is no eddy at all. For very great viscosities, we observe paste effects. The intermediate values offer the whole range of more or less fluid behaviours. In some of our simulations, made with low viscosities, the fluid divides into two or three smaller eddies within the mixing layer. More or less laminar fluids can be obtained depending on the radius of the viscous interactions in comparison with the inter-particle distances. In these fluids laminar layers can easily be distinguished from coexisting turbulent layers.

\section{Introducing pressure by adding elastic components-}

\subsection{Why and how to introduce elasticity}

In fluids, two phenomena coexist. Viscosity for which shear forces are parallel to flow, and shocks for which the irrotational forces are perpendicular to the direction of convection. Moreover, the concurrent effects of collisions cause another emergent phenomenon : pressure, which intensifies agglomeration effects (superpressure) or diffusion (depression) and which causes the formation of a third characteristic profile of turbulent fluid behaviour, that is Von Karman eddies. Now the question is : how to introduce pressure in a punctual mass model.

\subsection{Compressed elasiticity model}

Local pressure can be modelled by adding a thresholded elastic interaction between the particles of the same jet and between the particles of different jets. In the same way as for viscosity, we can say that pressure is an emergent phenomenon and we must try to model it without necessarily getting down to the scale of molecular thermal motion. Therefore we establish between the particles a thresholded elastic interaction with a low intensity and a large threshold in comparison with the threshold of the above viscous interaction. This is what we call a compressed elasticity model. 

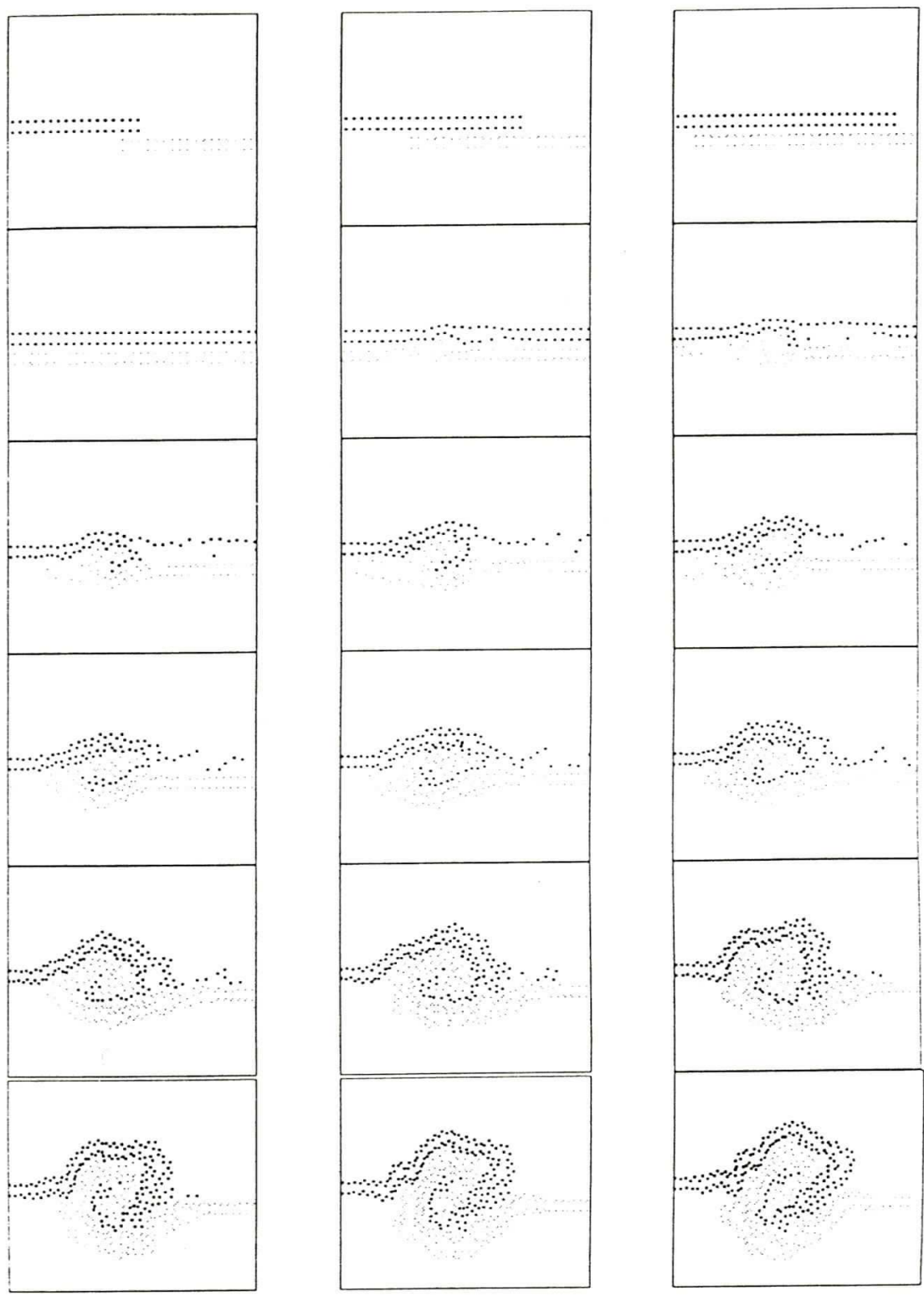

figure 9 Double-layer whirlpool. The time step of this chronogram is $1 \mathrm{sec}$ 

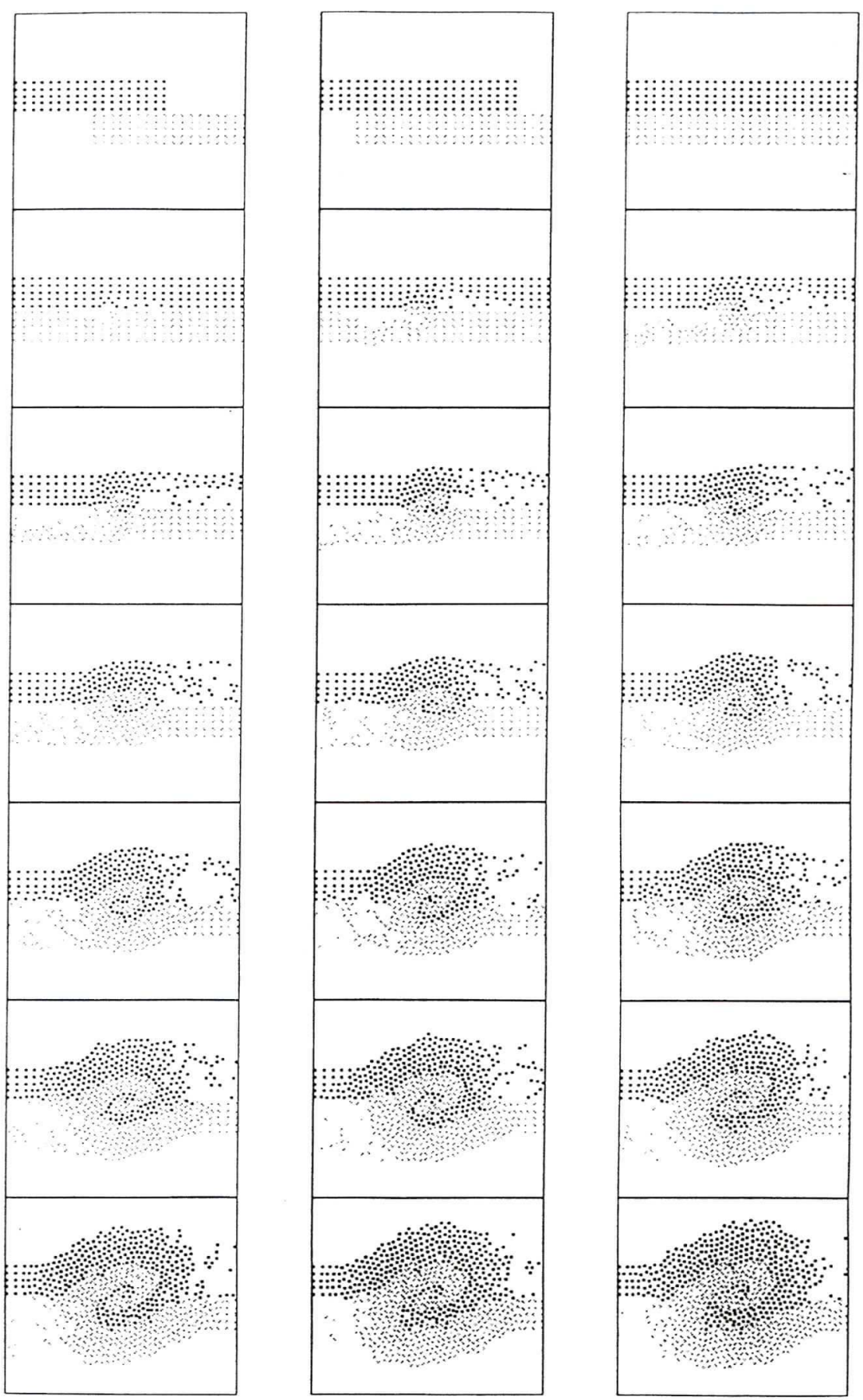

figure 10 Multi-layer whirlpool. The time step of this chronogram is $1 \mathrm{sec}$ 
This elastic repulsion force has a very low stiffness, and it only weakly prevents the particles from entering the neighbouring particles' high-viscosity zones. When the particles are linked by viscous interactions, they are in a confined state. Since the threshold is large, the repulsion between the masses is not caused by proper individual collisions. It is integrated on a neighbourhood through the overlapping of the elastic interaction zones. Thus each particle is in repulsive interaction with several neighbouring particles (about 15, in our simulations)(figure 11).
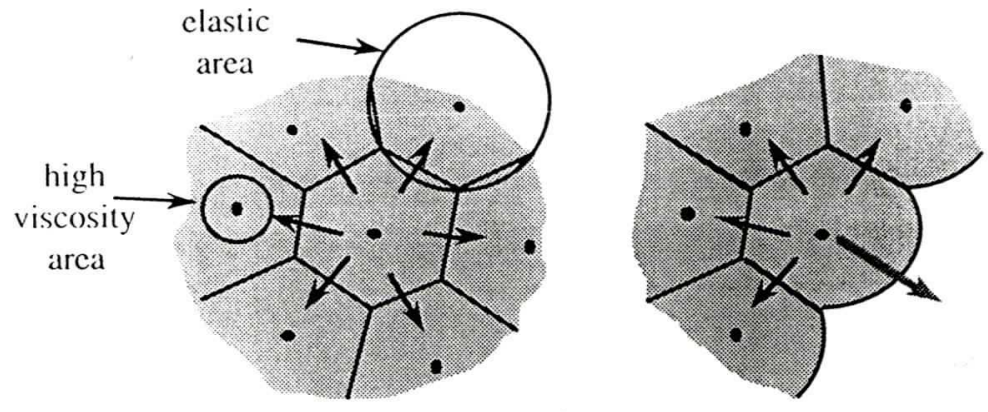

figure 11 Model of pressure

\subsection{Global confinement}

The introduction of a repulsion force requires a confined system. Such a confinement can be modelled by setting a visco-elastic buffer interaction with external masses acting as walls. This interaction must not be too stiff, in order to avoid the occurrence of shock waves from the walls. Fairly loose elastic buffer interactions enable us to model external pressure instead of rigid walls.

\subsection{Results}

Figure 12 shows the depression that appears behind an obstacle. The obstacle is represented by a visco-elastic buffer interaction.

We also obtained elastic oscillation effects propagating on laminar flows. (figure 13)

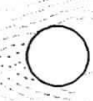

figure 12 Depression behind an obstacle
If one of the jets is replaced by a set of particles scattered over the simulation space we obtain the major effects that appear in a fluid flowing in a medium. Figure 14 shows the shock wave effects in the medium. Figure 15 shows von Karman profiles with inversed KelvinHelmholtz eddies.

Thus smoke behaviour has been modelled, including all the phenomena mentioned above : laminar flow, whirlpools, Von Karman eddies, dissipation (figure 16) 


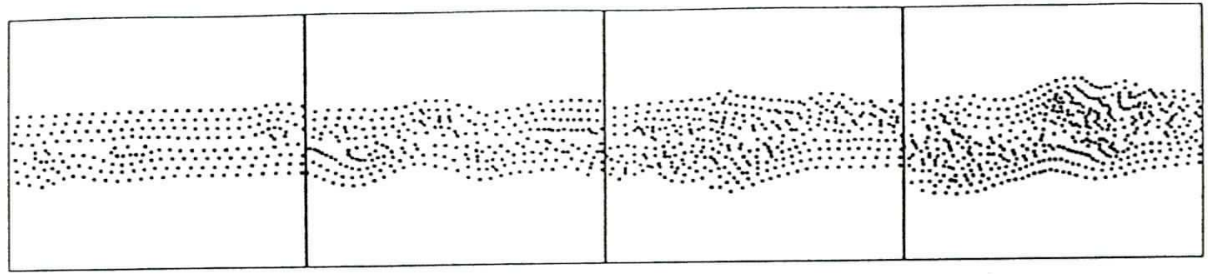

figure 13 Propagation (the time step of this chronogram is $1 \mathrm{sec}$ )
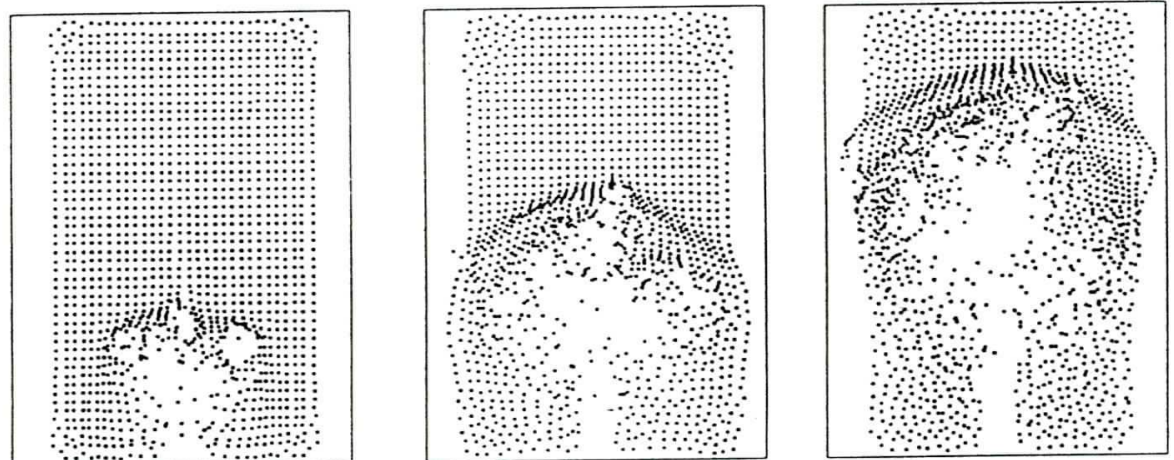

figure 14 Frontal shock with the jet and the medium

\section{Refinement}

5.1. Principles : physically-based refinement

When the motion of the particles corresponds to a correct motion, each of these particles has to be associated with an elementary volume in order that the combination of all these volumes forms the global volume of a specific turbulent material.

In accordance with the high deformability of this type of material, we know that this volume must also be highly deformable. Furthermore, the

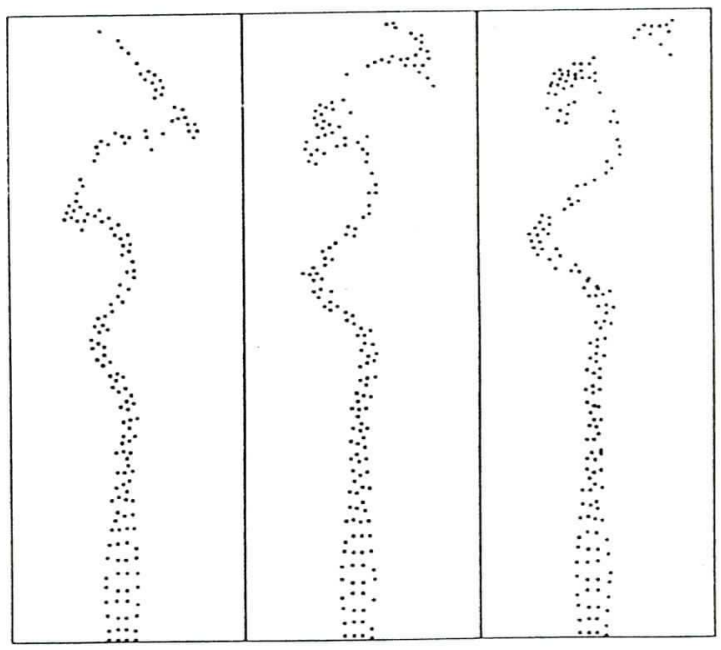

figure 15 Von Karman eddies in smoke deformation of this elementary volume cannot be arbitrarily defined. It must correspond to the physical properties of the modelled phenomenon and it cannot be independent of the movement of the particle.

We consider the dynamic phenomena involved in turbulent materials as the combination of a global dynamic behaviour (the behaviour of the particles) and a local dynamic behaviour (the behaviour of the particles' shape) This is the reason why, our refinement model is a physical model. 


\subsection{Multi-scale modelling}

Our hypothesis is that the local dynamic deformations involve low energies. Therefore they are influenced by the global dynamic behaviour but the converse is not true. Thus the final animation is obtained by coupling the particle model with a refinement model that will add the finer deformations to the sequence.

The fact that we work with two separate physical models enables us to simulate each of them at its own simulation rate, with its own resolution and according to its proper specific requirements. A rather small number of particles (300 to 1500) seems to be sufficient for the observation of phenomena such as curls, eddies, Kelvin-Helmholtz and Von Karman turbulences.

In order to obtain smooth wisps of smoke, a far higher resolution is required, but the properties of this model may be much simpler since the turbulences have already been obtained. This is called multi-scale modelling [LHM 95] and it makes it possible to produce quality motions at reasonable costs.

\subsection{The refinement model}

Our refinement model is a linear physical model already described in a previous publication [LHM 95]

Refinement is performed by a deformation field DF. Each point $\mathrm{M}_{\mathrm{ijk}}$ of the simulated space is characterized by a scalar dynamic variable called the deformation value $a_{i j k}(t)$. The whole scene is characterized by a deformation field $\mathrm{DF}(\mathrm{t})$. The particles passing through the scene deform this field. What we represent on the final image is not the particles but their zone of influence $\mathrm{ZI}(\mathrm{t})$ on the deformation field $\mathrm{DF}(\mathrm{t})$, that is, the set of points for which the deformation value is greater than a specific threshold value. In this work, the light intensity of each point is proportional to its deformation value. The zone of influence $\mathrm{ZI}(\mathrm{P}, \mathrm{t})$ of particle $\mathrm{P}$ is the elementary volume $\mathrm{V}(\mathrm{P}, \mathrm{t})$ that we associate with each particle ( $\mathrm{Cf} \S 5.1$ ). This is very comparable with the implicit surface methods.[Blinn 82][BS 91] The novelty consists in the way in which the particles modify the deformation field. We perform physical refinement by endowing this field with physical properties: The deformation value of each point varies as the position of a punctual mass with one degree of freedom (a phyxel). This mass is attached to a fixed substratum by a visco-elastic interaction and to its neighbouring masses by other visco-elastic interactions. Therefore The deformation field DF returns to a specific rest value after each transient deformation.

Thus, the volume $\mathrm{S}(\mathrm{P})$ depends on the motion of the particle. For example, the zone of influence of a particle at rest is a sphere with gradations and a blur, but the ZI of a moving particle has also a "comet's tail" whose size, direction and intensity distribution depends on the particle's velocity and trajectory. In addition, a group of close particles is visualized as a blurred continuous stretch of matter (figure 16).

\section{Conclusions}

Our final particle model (sections 3 and 4): (1) is at a larger scale than the molecular scale and models emergent effects such as viscosity and pressure with punctual masses in interaction; (2) integrates cohesion forces (viscous forces) that cause eddies, and repulsion forces that cause superpressure and depression, that intensify confinement or the fluid's dispersion and that also cause shock wave propagation, (3) properly 
reproduces the major characteristic figures of turbulent fluids such as plain curls, Kelvin-Helmholtz eddies and Von Karman wakes. Thus we have modelled various behaviours of turbulent objects : more or less viscous pastes, fluids, gasses, smokes.
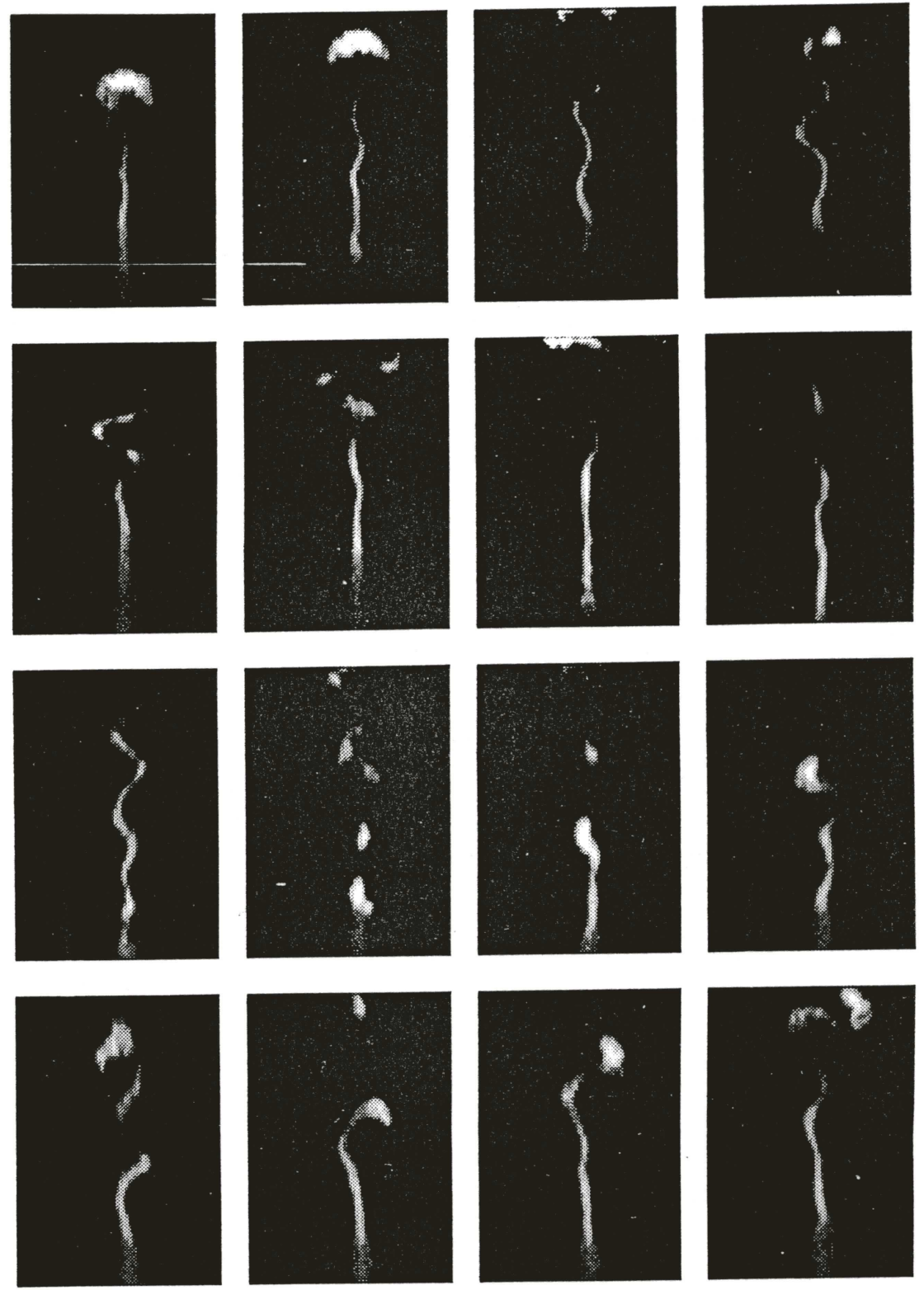

figure 16 A refined model of smoke. The time step of this chronogram is $1 \mathrm{sec}$

Moreover, the dynamic model constructed by the Cordis-Anima formalism, which is based on point physics and interactions makes it possible, at any instant, to act upon 
any given mass (respectively interaction automaton) in the system. This action may consist in the application of forces (respectively positions) of the external environment. Thus the operator or an object of the real world can act upon the simulated fluids without affecting the physical consistence of the system.

These simulations were carried out at $1050 \mathrm{~Hz}$. The refinement model is a finely discretized 1D linear $O(n)$ model. The final results are displayed on the chronogram on figure 16. These refinement simulations, with 400 smoke particles and 57600 phyxels, at the simulation frequency of $300 \mathrm{~Hz}$ required 7.06 seconds per frame on a Silicon Graphics Indigo 2 Extreme. However, this refinement model can easily be parallelized and implemented on a massively parallel machine.

\section{References}

[Gre 73] D. Greenspan Discrete Models, Reading in Applied Mathematics, Addison Wesley, 1973

[MP 89] G. Miller, A. Pearce Globular Dynamics : A Connected Particle System For Animating Viscous Fluids, Computer \& Graphics Vol 13, No. 3, pp 305-309 1989

[LJCFR91] A. Luciani, S. Jimenez, C. Cadoz, J.L. Florens, O. Raoult Computational Physics : a Modeler - Simulator-for Animated Physical Objects, Proceedings of Eurographics Conference, 1991, Vienna, Austria

[WH 91] J. Wejchert, D. Haumann Animation Aerodynamics In Proceedings of SIGGRAPH' 91 Volume 25, Number 4, July 1991

[CMTM 94] N. Chiba K. Muraoka H. Takahashi and M. Miura Two-dimensional Visual Simulation of Flames, Smokes and the Spread of Fire. The Journal of Visualization and computer Animation, vol 5, pp 37-53, 1994.

[PP 94] LC. H. Perry, R.W. Picard Synthesizing Flames and their Spreading Proceedings of the Fifth Eurographics Animation and Simulation Workshop (Oslo,

[SF 93] J. Stam and E. Fiume Turbulent Wind Fields for Gaseous Phenomena ACM Computer Graphics (SIGGRAPH' 93), p 369-376, August 1993

Norway September 17-18 1994)

[INA 90] M. Inakage A Simple Model of Flames Proceedings of CG International'90, pp. $71-81$

[LHM 95] A. Luciani, A. Habibi, E. Manzotti A Multi-Scale Physical Model of Granular Materials Proceedings of Graphics Interface' 95.

[CLF 93] C. Cadoz, A. Luciani, J.L. Florens CORDIS-ANIMA A Modelling and Simulation System for Sound and Image Synthesis - The General Formalism, Computer Music Journal, 1993, 10(1), 19-29, M.I.T. Press

[Les 94] M. Lesieur La Turbulence Presses Universitaires de Grenoble 1994

[Gre 73] D. Greenspan Discrete Models Addison-Wesley Reading M.A. 1973

[Blinn 82] J.F. Blinn A Generalization of Algebraic Surface Drawing ACM Transactions on Graphics Vol. 1, No.3, July 1982, Pages 235 - 256

[BS 91] J Bloomenthal, K. Shoemake Convolution Surfaces Proceedings of SIGGRAPH'91 Computer Graphics Volume 25, Number 4, July 1991

\section{Acknowledgements}

Thanks to Jean Loup Florens (ACROE) and Benoît Chanclou (LIFIA) who advanced the primary ideas of this work. Parts of this work have been used for an artistic work called ESQUISSES created for the IJCAI conference on August $31^{\text {st }} 1993$

The financial assistance of the French "Ministère de la Culture", the "Centre National Cinématographique", and the IMAG Institute is gratefully acknowledged. 